\title{
Rinoplastia endonasal: um estudo do grau de satisfação dos pacientes em cinco anos
}

\author{
Endonasal rhinoplasty: a study of patient satisfaction in five years
}

\begin{abstract}
Ewaldo Bolivar de Souza
PINTO $^{1}$

Rachid Gorron MaLOOF ${ }^{2}$

Rodrigo Thiago Dutra ${ }^{2}$

Alexandre Sanfurgo DE

CARVALHO $^{2}$

Luis Humberto URibe

MORELli $^{3}$

Fernanda Provazi Zanetta ${ }^{4}$

Rogério Porto da Rocha ${ }^{5}$

Sandra Márcia da Silva

Mó́A ${ }^{5}$

Priscila C. S. P. AbDalla ${ }^{5}$
\end{abstract}

Trabalho realizado na Clínica Dr. Ewaldo Bolívar de Souza Pinto, Santos, SP, Brasil.

Artigo submetido pelo SGP (Sistema de Gestão de Publicações) da RBCP.

Artigo recebido: 29/9/2010 Artigo aceito: 5/11/2010

\begin{abstract}
RESUMO
Introdução: Muito tem sido discutido a respeito das várias técnicas e vias de acesso em rinoplastia. Todas as técnicas buscam um resultado previsível e reprodutível, associado à melhora estética e funcional do nariz. Nesse trabalho, foi avaliado o nível de satisfação dos pacientes submetidos à rinoplastia nos últimos cinco anos: rinoplastia reducional por via endonasal associada ou não a rinodinâmica. Método: Por meio de um estudo retrospectivo foram identificados 201 pacientes submetidos à rinoplastia endonasal entre 2005 e 2010. Todos os procedimentos foram realizados com a mesma rotina cirúrgica, conforme indicação. Os pacientes foram avaliados de maneira objetiva quanto à cirurgia realizada e, de maneira subjetiva, a respeito de sua função respiratória anterior e posterior à intervenção cirúrgica e o grau de satisfação quanto à estética nasal por meio de um questionário. Resultados: A idade dos pacientes variou de 14 a 68 anos; com predominância da raça branca $(96,75 \%)$. Referiram melhora no padrão respiratório após a cirurgia, $83,87 \%$ dos pacientes estudados. Em relação à estética nasal, $30(32,26 \%)$ pacientes tiveram suas expectativas superadas, 42 $(45,16 \%)$ consideraram muito boa, $18(19,36 \%)$, como boa e três $(3,22 \%)$, como abaixo do esperado. Conclusão: O procedimento resultou em melhora da função respiratória em quase $90 \%$ dos pacientes, além disso, o alto grau de satisfação com a estética exposto pelos pacientes permite concluir que rinoplastia endonasal tem apresentado ótimos resultados até o momento.
\end{abstract}

Descritores: Rinoplastia/métodos. Satisfação do paciente. Respiração. Estética.

\begin{abstract}
Background: Much has been discussed about the various techniques and access routes in rhinoplasty. All seek a predictable and reproducible associated with improved aesthetic and functional nose. This study evaluated the level of satisfaction of patients undergoing rhinoplasty in the past five years: by endonasal reductional rhinoplasty associated or not to rhino dynamic. Methods: Through a retrospective study identified 201 patients who underwent endonasal rhinoplasty the period between 2005 and 2010. All procedures were performed with the same routine as surgical indication. Were evaluated in an objective manner about the surgery and subjectively about their lung function before and after surgery and the degree of satisfaction with the nasal aesthetics through a questionnaire. Results: The age ranged between 14 and 68 years of age; predominant among Caucasians (96.75\%). 83.87\% of the patients changed their minds after surgery, indicating improvement in breathing pattern. Regarding the nasal aesthetic, $30(32.26 \%)$ patients had exceeded their expectations, 42 $(45.16 \%)$ felt very good, $18(19.36 \%)$ looked as good and three $(3.22 \%)$ evaluated with lower than expected. Conclusion: The high degree of satisfaction with the aesthetics espoused by patients as well as lead to an improvement of respiratory function in $90 \%$ notes that the procedure performed has great result so far.
\end{abstract}

Keywords: Rhinoplasty/methods. Patient satisfaction. Respiration. Esthetics.

1. Chefe do Serviço de Cirurgia Plástica "Dr. Ewaldo Bolívar de Souza Pinto" - UNISANTA, Santos, SP, Brasil. Membro Titular da Sociedade Brasileira de Cirurgia Plástica (SBCP), Ex-presidente da SBCP.

2. Cirurgião Geral; Médico Residente de $3^{\circ}$ ano do Serviço de Cirurgia Plástica "Dr. Ewaldo Bolívar de Souza Pinto" - UNISANTA, Santos, SP, Brasil.

3. Cirurgião Geral; Médico Residente de $1^{\circ}$ ano do Serviço de Cirurgia Plástica "Dr. Ewaldo Bolívar de Souza Pinto" - UNISANTA, Santos, SP, Brasil.

4. Cirurgiã Geral; Médica Estagiária do Serviço de Cirurgia Plástica "Dr. Ewaldo Bolívar de Souza Pinto" - UNISANTA, Santos, SP, Brasil.

5. Cirurgião Plástico, Membro Especialista da SBCP, Preceptor do Serviço de Cirurgia Plástica "Dr. Ewaldo Bolívar de Souza Pinto" - UNISANTA, Santos, SP, Brasil. 


\section{INTRODUÇÃO}

Muito tem sido discutido a respeito das várias técnicas e vias de acesso em rinoplastia ${ }^{1-4}$. A rinoplastia reducional (ou tradicional), estrutural, a endorinoplastia e a exorinoplastia todas buscam um resultado previsível e reprodutível, associado à melhora estética e funcional do nariz do paciente ${ }^{5-7}$.

É necessária a observação dos resultados em longo prazo para identificar se o objetivo foi alcançado ou não. Nesse trabalho foi avaliado o nível de satisfação individual dos pacientes submetidos à rinoplastia desempenhada por único cirurgião nos últimos cinco anos: rinoplastia reducional por via endonasal associada ou não a rinodinâmica.

A rinodinâmica surgiu em 1993, após estudo anatômico do músculo depressor do septo (MDS) e sua relação com o terço distal do nariz em cadáveres ${ }^{8-10}$. A técnica consiste na liberação e/ou secção da inserção dos fascículos mediais do músculo depressor do septo (da maxila) e plicatura dos fascículos intermédios com sepultamento do coto muscular dos fascículos mediais. Isto promove o aumento do ângulo nasolabial, com elevação da ponta nasal. A via de acesso é realizada pela mucosa do lábio superior em forma de " $\mathrm{Z}$ " (sendo o braço principal o freio do lábio superior), podendo ainda ser realizada rotação dos retalhos, conforme a indicação ${ }^{11-14}$.

\section{MÉTODO}

Por meio de um estudo retrospectivo baseado em revisão de prontuários do Serviço de Cirurgia Plástica do UNISANTA - Santos, SP foram identificados 201 pacientes submetidos à rinoplastia endonasal, realizadas pelo autor principal entre o período de janeiro de 2005 e janeiro de 2010.

Por meio de ligações telefônicas diretas e/ou e-mail, tentou-se a localização dos pacientes. Dentre os 201 pacientes, $108(53,7 \%)$ não foram contatados ou não quiseram participar do estudo. Os 93 (46,3\%) pacientes que aceitaram participar do estudo foram avaliados de maneira objetiva sobre a cirurgia realizada e, de maneira subjetiva, a respeito de sua função respiratória anterior e posterior à intervenção cirúrgica, além do grau de satisfação quanto à estética nasal por meio de um questionário (Figura 1).

Todos os procedimentos foram realizados seguindo a mesma rotina cirúrgica, conforme indicação: rinodinâmica, tratamento do septo nasal, rinoplastia por via endonasal (conforme descrito por Joseph, com incisão intercartilaginosa), osteotomia e enxertia de cartilagem septal.

A satisfação com a estética nasal geral foi avaliada em graus, sendo considerado: abaixo do esperado, boa, muito boa e além do esperado. A avaliação subjetiva dos pacientes foi agrupada para a identificação dos aspectos funcionais e estéticos do resultado cirúrgico.

\begin{tabular}{|c|}
\hline Questionário de Avaliação \\
\hline Nome \\
\hline Data da Cirurgia __ \\
\hline QUANTO À RINOPLASTIA \\
\hline 1- É a primeira vez que opera o nariz? \\
\hline Sim___ Não___ Quantas vezes? \\
\hline 2- Caso tenha operado mais de uma vez, todas foram com o Dr. Ewaldo \\
\hline Bolivar? \\
\hline Sim__ Não_ \\
\hline 3- Foi realizada rinodinâmica? \\
\hline Sim__ Não___ \\
\hline 4- Foi realizado o tratamento do septo nasal? \\
\hline Sim__ Não__ \\
\hline 5- Foi realizado enxerto de cartilagem? \\
\hline Sim__ Não___ Onde? \\
\hline 6- Foi realizada osteotomia (fratura óssea) na cirurgia? \\
\hline Sim__ Não__ \\
\hline 7- Após a cirurgia teve alguma complicação? \\
\hline Sim__ Não___ Qual? \\
\hline 8- Foi necessário algum tratamento? \\
\hline Sim__ Não___ \\
\hline 9- Foi necessária uma nova cirurgia? \\
\hline Sim___ Não____ \\
\hline QUANTO À FUNÇÃO RESPIRATÓRIA \\
\hline 10- Antes da cirurgia, você achava que respirava normal? \\
\hline Sim_ Não___ \\
\hline 11- E depois da cirurgia, você mantém a sua opinião da resposta anterior? \\
\hline Sim__ Não___ \\
\hline 12- Hoje você respira com dificuldade? \\
\hline Sim__ Não___ Melhor? \\
\hline
\end{tabular}

Figura 1 - Questionário de avaliação.

\section{RESULTADOS}

O estudo identificou 93 pacientes, sendo 72 do sexo feminino e 21 do sexo masculino, sendo a relação homem: mulher de 1:3,43. A idade variou entre 14 anos e 68 anos, com idade média de 28,3 anos. Predominaram os pacientes da raça branca $(96,75 \%)$ e da negra, $3,25 \%$. Foram realizados 102 procedimentos, sendo $93(91,18 \%)$ primários e nove $(8,82 \%)$ cirurgias secundárias, destes, seis $(5,88 \%)$ foram operados primariamente pelo autor, sendo considerados, então, como revisão.

As cirurgias foram realizaram de acordo com a indicação de cada paciente: 1) rinodinâmica em $84(90,32 \%)$ pacientes; 2) tratamento do septo nasal em $72(77,42 \%)$, 3) rinoplastia por via endonasal em $100 \%$ dos casos, 4) osteotomia em 42 $(45,16 \%)$ pacientes. Enxertia de cartilagem septal foi realizada em nove $(9,68 \%)$ pacientes, todos receberam enxerto de ponta nasal $(100 \%)$ e apenas dois $(22,23 \%)$ também receberam enxerto em dorso. 
No período pré-cirúrgico, apenas seis $(6,45 \%)$ pacientes se queixavam de algum tipo de dificuldade respiratória. No entanto, $78(83,87 \%)$ pacientes mudaram de opinião após a cirurgia, referindo melhora no padrão respiratório e seis $(6,45 \%)$, apesar da cirurgia, apresentaram algum grau dificuldade para respirar.

Quanto aos resultados estéticos, as apreciações positivas revelam-se em sua maioria. Em relação à estética nasal, 30 $(32,26 \%)$ pacientes tiveram suas expectativas superadas, 42 $(45,16 \%)$ consideraram muito boa, $18(19,36 \%)$ analisaram como boa e três $(3,22 \%)$ como abaixo do esperado.

No tocante às mudanças na forma nasal, nove $(9,67 \%)$ pacientes notaram pouca alteração, 30 (32,26\%), como moderada, e $54(58,07 \%)$ consideraram que houve muita alteração. Nenhum paciente deixou de perceber algum tipo de mudança. No tangente à relação face-nariz, sua avaliação foi sempre positiva, com $24(25,80 \%)$ pacientes referindo como boa a nova relação, 39 (41,93\%), como muito boa, e 30 (32,26\%), como além do esperado.

O grau de satisfação foi avaliado com quatro itens: abaixo do esperado por três $(3,22 \%)$ pacientes; boa por seis $(6,45 \%)$; muito boa por $33(35,47 \%)$ e $51(54,83 \%)$ referiam estar satisfeitos além do esperado com o resultado. As Figuras 2 a 4 ilustram alguns casos desse estudo.

\section{DISCUSSÃO}

A procura pela rinoplastia é norteada por razões estéticas. $\mathrm{O}$ desconhecimento dos pacientes sobre a sua qualidade
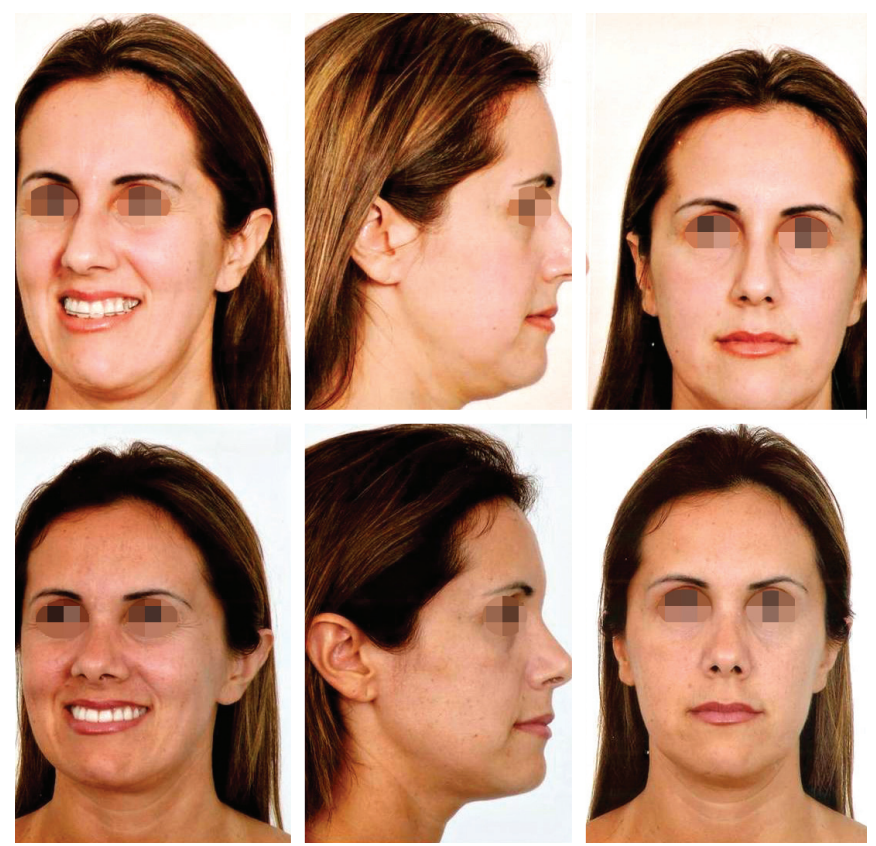

Figura 2 - Pré e pós-operatório de 5 meses.
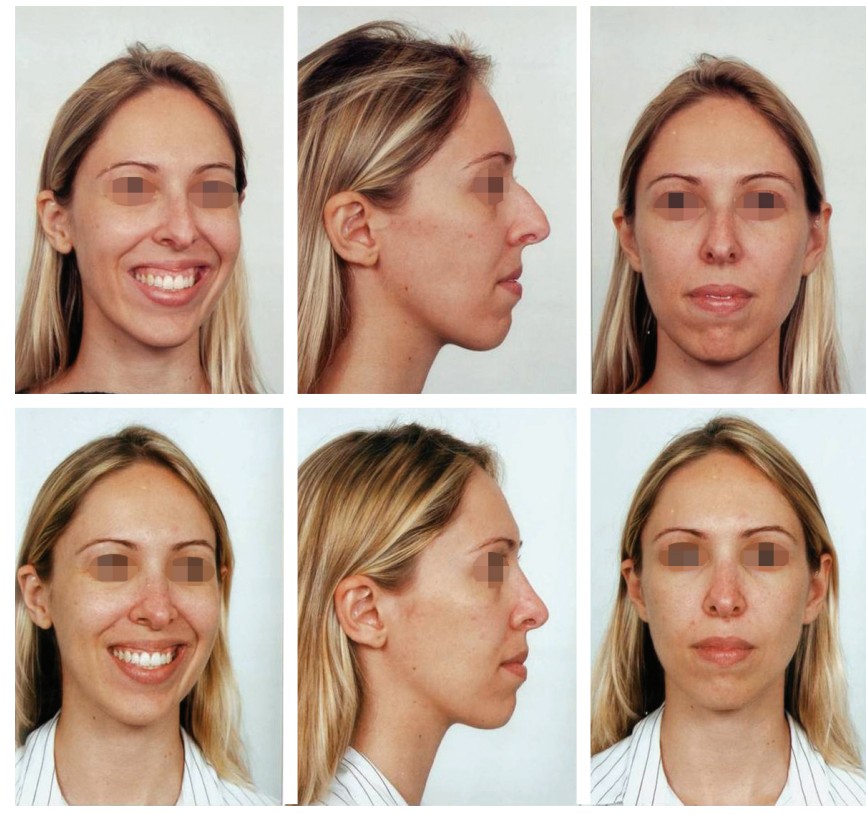

Figura 3 - Pré e pós-operatórios de 7 meses.
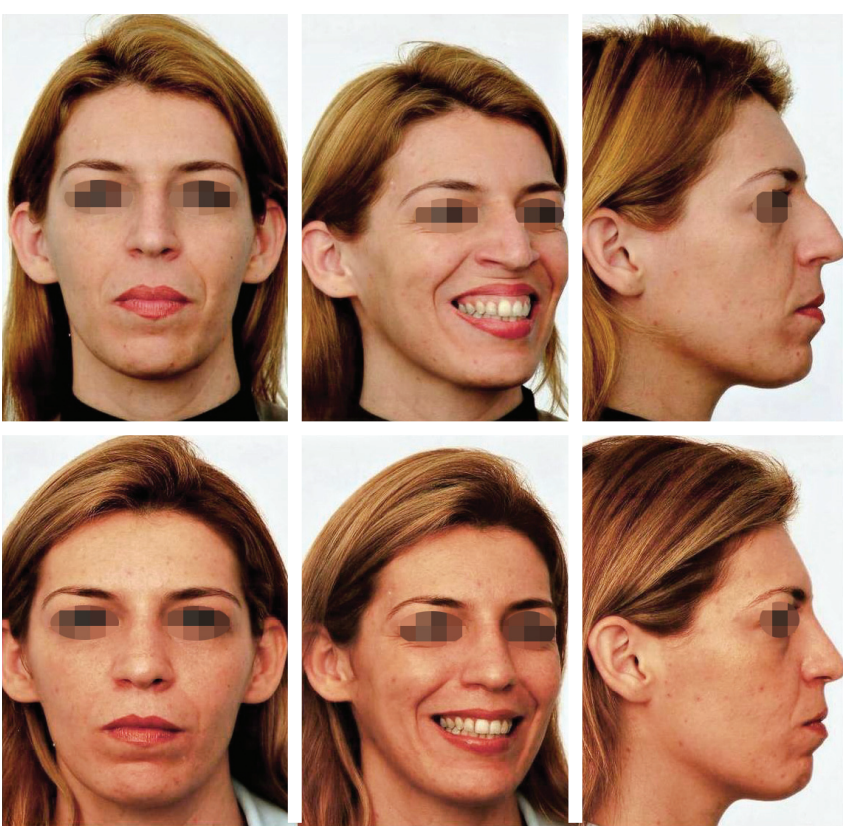

Figura 4-Pré e pós-operatórios de 10 meses.

funcional respiratória $(83,87 \%)$ nos faz responsáveis pelo diagnóstico e tratamento adequado. O binômio beleza/ função, em especial na rinoplastia, é essencial para que o resultado seja admirável e saudável.

Nessa análise de dados, o cirurgião conseguiu, através de sua abordagem, melhora da estética nasal $(96,78 \%)$ e da sua relação com a face $(100 \%)$, causando modificações perceptíveis em 84 pacientes, combinado com a otimização do padrão respiratório em 78 deles. 
Dos três resultados desfavoráveis em relação à estética, nenhum foi relacionado com piora do padrão respiratório. E os seis pacientes que apresentavam alteração funcional anterior à cirurgia mantiveram a queixa com o mesmo ou menor grau. Esta evidência demonstra que a rinoplastia reducional não tem um caráter iatrogênico, independentemente dos resultados, favoráveis ou não.

Cabe aqui lembrar que este estudo foi realizado avaliandose apenas o resultado da rinoplastia reducional com rinodinâmica executada pelo mesmo cirurgião, e assim, não questiona se o fato do procedimento for melhor ou não aos outros tipos de abordagens. Esse mesmo resultado pode não ser atingido por cirurgiões inexperientes.

\section{CONCLUSÃO}

O alto grau de satisfação com a estética exposto pelos pacientes $(96,78 \%)$, além de conduzir a uma melhora da função respiratória em quase $90 \%$, constata que o procedimento realizado tem ótimo resultado até o momento.

A análise dos dados por meio dos relatos dos pacientes selecionados demonstrou um ótimo padrão de qualidade obtido pela intervenção desenvolvida. Ou seja, a rinoplastia tradicional ou reducional associada à rinodinâmica obteve ótimos resultados estético-funcionais, não sendo iatrogênica em mãos experientes.

Este estudo científico, devido a sua natureza subjetiva por meio de pontos de apreciação ou não do resultado estético e funcional e sua comparação entre o pré e póscirúrgico individualizado por paciente, pode ser válido, uma vez que as informações colhidas de um grande grupo podem apoiar interpretações e impressões clínicas.

\section{REFERÊNCIAS}

1. Joseph J. Nasenplastik um sonstige gesechtsplastik, webst einem anhang uber mammaplastik und einige weitere operationen aus dem gebiete der ausseren Korperplastik; ein atlas und lehrbruch. Curt kabitzch. Leipzig;1931

2. Gonella HA. Contribuição ao estudo anatômico dos músculos do nariz [tese]. Sorocaba:PUCSP;1982.

3. Sheen JH. Aesthetic rhinoplasty. St. Louis: CV Mosby;1987.

4. McCarthy JG, Wood-Smith D. Cirugía plástica. La cara. Tomo II. Buenos Aires:Editorial Medica Panamericana;1992.

5. Pitanguy I, Ferreira G, Amorim N. Importancia del ligamento dermocartilaginoso en la rinoplastia. In: Coiffman F, ed. Cirugía estética y reconstructiva de la cara y el cuello. Tomo II. $3^{\mathrm{a}}$ ed. Colombia:AMOLCA;2007. p.1573.

6. Papadopulos EA, Papadopulos CA. Rinoplastia y rinoseptoplastia. In: Coiffman F, ed. Cirugía estética y reconstructiva de la cara y el cuello. Tomo II. $3^{\mathrm{a}}$ ed. Colombia:AMOLCA;2007. p.1537.

7. Bohorquez EC. Anatomia quirurgica de la nariz. In: Coiffman F, ed. Cirugía estética y reconstructiva de la cara y el cuello. Tomo II. $3^{\mathrm{a}}$ ed. Colombia:AMOLCA;2007. p.1533.

8. De Souza Pinto EB, Erazo IP, Queiroz FW. Rhinoplasty: treatment of the tip-columella and lip. Annals of the ISAPS - XIII International congress, New York, September 28 - October 3, 1995.

9. De Souza Pinto EB, Erazo IP, Muniz AC. The nasal tip surgical treatment by performing the nasal septum depressor muscle miopalsty. Abstracts of the Fourteenth Congress International Society of Aesthetic Plastic Surgery - São Paulo -Brazil, May 31 - June 3, 1997: 245.

10. De Souza Pinto EB, Rocha RP, Queiroz Filho W, Spada Neto E, Zacharias KG, Amâncio A, et al. Anatomy of the mediam part of the septum depressor muscle in aesthetic surgery. Aesthetic Plast Surg. 1988;22(2):111-5.

11. De Souza Pinto EB, MunizAC, Erazo IP,Abdalla PC. Dynamic rhinoplasty: treatment of the tip muscles. Perspect Plast Surg. 1999;12(1):21-44.

12. Rohrich RJ, Huynh B, Muzaffar AR, Adams WP Jr, Robinson JB Jr. Importance of the depressor septi nasi muscle in rhinoplasty: anatomic study and clinical application. Plast Recontr Surg. 2000;105(1):376-83.

13. De Souza Pinto EB. Relationship between tip nasal muscles and the short upper lip. Aesthetic Plast Surg. 2003;27(5):381-7.

14. Rohrich RJ, Muzaffar AR, Janis JE. Component dorsal hump reduction: the importance of maintaining dorsal aesthetic lines in rhinoplasty. Plast Reconstr Surg. 2004;114(5):1298-308. 